\title{
Pricing and ordering decisions of risk-averse newsvendors: Expectile-based value at risk (E-VaR) approach
}

\author{
Hande Gunay Akdemir \\ Giresun University, Department of Mathematics, Faculty of Arts and Science, Giresun, Turkey
}

Received: 21 November 2017, Accepted: 11 February 2018

Published online: 7 April 2018.

\begin{abstract}
In this study, we investigate optimal pricing and ordering decisions based on different levels of risk aversity. By using E-VaR measure as an alternative to expectation operator, a one-parameter extension of the classical price-setting newsvendor model is obtained. For the additive demand model, a simulation study is conducted to compare optimal prices and orders of risk averse newsvendors with those of less prudent and risk taker ones.
\end{abstract}

Keywords: Joint pricing and inventory decisions, newsvendor model, price sensitive stochastic demand, risk measures, risk behaviors.

\section{Introduction}

We consider a newsvendor model in which a risk averse decision maker (DM) simultaneously decides the selling price and order quantity. Depending on the different risk attitudes of the DMs, the optimal price and order deviate from the risk-neutral optimal decisions that maximize the total expected profit. A risk-averse DM aims to find the optimal balance between the total overage and underage costs with low volatility in his objective which leads him to be prudent against substantial losses, for more detail see [30]. Inventory problems are similar to investment problems in finance since decisions have to be taken in a stochastic environment, and risk preferences of the DMs are important to include, see [13]. For this reason, the processes performed in financial risk management are also useful for inventory problems. We focus on three basic distribution-based risk measures: Value at Risk (VaR), Conditional Value at Risk (CVaR) and EVaR which was defined by Kuan et al. [19]. VaR and CVaR are quantile-based measures for quantification of financial risk, and they have also been used in stochastic inventory decision problems for controlling profit (or loss) variability. Despite its wide applicability and simplicity, some criticisms have been done on VaR, such as not being subadditive that causes penalization of diversification, being difficult to optimize (neither convex nor concave), and insensitive to extreme losses that occur with small probability, for a detailed analysis see [22]. On the other hand, coherent risk measures (such as CVaR) have ideal behavioral properties, namely monotonicity, translation invariance, positive homogeneity and subadditivity (see [4]). Newey and Powell [20] introduced expectiles which are known to be coherent risk measures. Typically, as a family of generalized quantiles, expectiles are more concentrated around the mean than the corresponding quantiles (see [5]). Besides, they are more sensitive to the size of extreme losses relatively to VaR since they rely on more comprehensive information (see [19]). Moreover, expectiles are suitable for forecasting, backtesting and bayesian decision making (see [15]).

Coherent risk measures have commonly used in newsvendor models as an alternative to the expectation operator in order to take into account risk preferences. The literature that deals with risk by using VaR and CVaR, but does not consider 
pricing as a decision, is as follows. Jammernegg and Kischka [17] provided profit maximizing newsvendor solutions with risk considerations by using mean-CVaR criterion. Gotoh and Takano [16] adopted both single and multiple item newsvendor models for the minimization of convex mean-CVaR objectives for two types of loss functions, namely the net loss and the total cost. The latter considered positive shortage cost, while the former considered no shortage cost. Ahmed et al. [1] analyzed the extension of the classical multi-period newsvendor model considering coherent risk measure objective. In [9] and [10], the authors studied mean-risk models with law-invariant coherent risk measures in risk averse newsvendor problems. Özler et al. [21] utilized VaR as the risk measure in a newsvendor framework and investigated two-product version under a VaR constraint. In [26] and [27], trade-off analyses between expected profit and CVaR were done via two risk parameters in risk-averse inventory models. Katariya et al. [18] showed that the impact of risk aversity on the optimal order quantities depends on the demand distribution and the cost parameters for CVaR criterion newsvendor problem. Wu et al. [23] investigated the newsvendor model with random shortage cost under CVaR criterion. Arikan and Fichtinger [3] studied the risk-averse newsvendor problem with spectral risk measure objective that covers many coherent risk measures including $\mathrm{CVaR}$ and mean-CVaR. The authors also argued the impact of both the monotonicity of the objective function and the selling price on risk neutral and risk averse optimal order quantities.

Now, let us review the relevant models in which the price is not exogenously given. In [7], the authors adopted CVaR as the objective function in order to find the optimal pricing and ordering decisions for both additive and multiplicative demand models. Chiu and Choi [8] derived the optimal joint pricing and stocking decisions of the VaR newsvendor under price-dependent demands. In case emergency purchase option is allowed, Xu [25] investigated the impacts of parameter changes on the optimal selling price and order quantity of risk sensitive newsvendors. Within a newsvendor framework, Arcelus et al. [2] evaluated the pricing and ordering policies to meet price-dependent stochastic demand by maximizing of the risk-adjusted expected profit. In [24], the authors discussed the effects of both risk averseness and competition on the newsvendor's optimal ordering and pricing strategies under the CVaR criterion. Dai and Meng [11] considered a risk-averse newsvendor making a joint decision on ordering, pricing and marketing by using CVaR as the decision criterion. For a literature review on periodic inventory systems, the reader may refer to [28] in which time-consistent coherent risk measures are used to study a risk-averse firm's inventory and price control activities.

In this paper, risk averse solutions are evaluated under the EVaR minimization criterion in the newsvendor setting. The rest of this paper is organized as follows. In the next section, the notation used for the classical price-setting newsvendor model is given. In Section 3, the definitions and some properties of VaR, CVaR, and EVaR measures are given. The proposed EVaR criterion price-setting newsvendor model is presented and analyzed its risk attitude relations in Section 4. Afterwards, a numerical example is given for exponential error distribution. The paper is concluded with some remarks in Section 5.

\section{The classical price-setting newsvendor model}

In this section, the notation used for the basic price-setting newsvendor model is given. At the beginning of the time period, DM does not know how much he can sale, and needs to decide both the order quantity $q$ and the unit selling price $p$ before price-dependent stochastic demand is realized. The unit purchase price of the product is $c$. For an underprediction case, unsatisfied demand is penalized with a shortage (stock out) value per unit $s$. Leftover inventory is sold in a secondary market with discounted salvage price per unit $v$ for an overforecast case. It is assumed that $v<c<p$ and $s>0$. Demand is denoted by $D(p, \varepsilon)$, where $\varepsilon$ is stochastic error term with known distribution function $F_{\varepsilon}($.$) . Then, the profit of the$ newsvendor can be written as

$$
\begin{aligned}
\pi(p, q) & =p \min \{q, D(p, \varepsilon)\}-c q-s \max \{D(p, \varepsilon)-q, 0\}+v \max \{q-D(p, \varepsilon), 0\} \\
& =(p-c) D(p, \varepsilon)-(c-v)[q-D(p, \varepsilon)]^{+}-(p+s-c)[D(p, \varepsilon)-q]^{+}
\end{aligned}
$$


where $[\cdot]^{+}=\max \{\cdot, 0\},(c-v)$ and $(p+s-c)$ are unit overage and underage costs, respectively. By introducing the uncertain loss function $L(p, q)=-\pi(p, q)$, we get

$$
L(p, q)=(c-v)[q-D(p, \varepsilon)]^{+}+(p+s-c)[D(p, \varepsilon)-q]^{+}-(p-c) D(p, \varepsilon) .
$$

In this study, we only consider the additive demand model, so $D(p, \varepsilon)=a-b p+\varepsilon$ where $a, b>0$. The expected loss is derived as:

$$
E[L(p, q)]=\int_{0}^{q-a+b p}((c-v) q-(p-v)(a-b p+t)) d F_{\varepsilon}(t)+\int_{q-a+b p}^{\infty}(s(a-b p+t)-(p+s-c) q) d F_{\varepsilon}(t)
$$

with error range $[0, \infty)$.

\section{Some risk measures and their properties}

In order to better understand the financial meaning of risk quantifying, the concept of risk measure was defined axiomatically via acceptability concept in [4]. A financial position $L>0$ is defined by an uncertain monetary variable representing possible losses (if a possible value of $L$ is negative, it means that it denotes a gain), and its risk $\rho(L)>0$ is considered as the additional capital requirement for an acceptable position. A financial position become acceptable by adding to the risk value $\operatorname{VaR}_{\alpha}(L)$, if we say that the probability of loss does not exceed $(1-\alpha)$ for a specified, sufficiently high, threshold $\alpha \in(0,1)$. By definition, it can be interpreted as "maximum loss which is not exceeded with a given high confidence level $\alpha$ ", so

$$
\operatorname{VaR}_{\alpha}(L)=\inf \{l \in \mathbb{R} \mid P(L \leq l) \geq \alpha\}
$$

which can be referred to the $\alpha$-quantile of the underlying loss distribution, that is, $\operatorname{VaR}_{\alpha}(L)=F_{L}^{-1}(\alpha)$. Typical confidence levels are $0.90,0.95$ or higher.

As an alternative measure of risk, CVaR answers the question: "What is the expected loss of $(1-\alpha) \times 100 \%$ worst losses?". If the distribution function $F_{L}($.$) is continuous, C V a R_{\alpha}(L)$ equals to the conditional expectation of loss when the $\operatorname{VaR}_{\alpha}(L)$ is exceeded, that is

$$
\operatorname{CVaR}_{\alpha}(L)=E\left[L \mid L \geq \operatorname{VaR}_{\alpha}(L)\right]=\frac{1}{1-\alpha} \int_{\alpha}^{1} F_{L}^{-1}(t) d t .
$$

Definition 1. (Coherency [4]): A risk measure $\rho$ satisfying the following axioms is said to be coherent.

(Translation invariance) $\rho(L+m)=\rho(L)-m$, for $m \in \mathbb{R}$, adding risk-free cash to a position reduce its risk by the same cash.

(Subadditivity) $\rho\left(L_{1}+L_{2}\right) \leq \rho\left(L_{1}\right)+\rho\left(L_{2}\right)$, diversification cannot increase risk.

(Positive homogeneity) $\rho(\lambda L)=\lambda \rho(L)$, for $\lambda>0$, increasing the size of the position scales its risk by the same factor.

(Monotonicity) $L_{1} \leq L_{2} \Longrightarrow \rho\left(L_{1}\right) \leq \rho\left(L_{2}\right)$, the greater the loss, the greater the risk.

There are many considerations in choosing the proper risk measure. VaR has been widely used in practice, but it is not a subadditive risk measure. By contrast, CVaR is coherent. For a coherent risk measure, relaxation of the axioms positive homogeneity and subadditivity as convexity axiom:

$$
\text { (Convexity) } \rho\left(\lambda L_{1}+(1-\lambda) L_{2}\right) \leq \lambda \rho\left(L_{1}\right)+(1-\lambda) \rho\left(L_{2}\right) \text { for } \lambda \in(0,1) \text {, }
$$

one can get convex measures of risk. Convex and coherent risk measures capture and reflect the impact of behavioral risk preferences. For a detailed information on risk measures and their properties, reader may refer to [14]. To describe 
risk behaviors in the decision problem, we utilize expectiles which also have appealing risk measurement properties. Expectiles have resemblance to quantiles, in fact, an $\omega$ - expectile is also an $\alpha$-quantile (see [12] for the relation). It is well-known that $\alpha$-quantile can be defined as the minimizer of the expectation of the following asymmetric, piecewise linear score (error), that is

$$
l^{*}=F_{L}^{-1}(\alpha)=\underset{l}{\arg \min } E\left[\alpha(L-l)^{+}+(1-\alpha)(L-l)^{-}\right]
$$

where $\alpha \in(0,1),(L-l)^{+}=\max \{L-l, 0\}$ and $(L-l)^{-}=\max \{l-L, 0\}$. Similarly, $\omega$-expectile can be defined as the minimizer of the expectation of the following asymmetric, piecewise quadratic score, so

$$
e_{\omega}(L)=\underset{l}{\arg \min } E\left[\omega\left((L-l)^{+}\right)^{2}+(1-\omega)\left((L-l)^{-}\right)^{2}\right]
$$

for $\omega \in(0,1)$. From the first order of optimality condition, the following identity holds

$$
\omega E\left[\left(L-e_{\omega}(L)\right)^{+}\right]=(1-\omega) E\left[\left(L-e_{\omega}(L)\right)^{-}\right]
$$

If we take $\omega=0.5$, we simply get $e_{\omega}(L)=E[L]$. The function $e_{\omega}(L)$ is continuous and strictly increasing with respect to $\omega$ (see [5]). When a positive value of the random variable $X$ is assumed as a gain, that is, $X=-L$, then $e_{\omega}(L)=-e_{1-\omega}(X)$. Note that, our $\omega$ corresponds $(1-\omega)$ when the random variable denotes a gain as in [6], [12], [19]. Now, let us define $\operatorname{EVaR}_{\omega}(L)=e_{\omega}(L)$. From (9), $\operatorname{EVaR}_{\omega}(L)$ can be seen as monetary value that should be added to the position in order to have for a specified, sufficiently high, gain-loss ratio $\frac{\omega}{1-\omega}$. In [19], $\omega$ is referred to as an index of prudentiality, and $\operatorname{EVaR}_{\omega}(L)$ is understood as the margin (capital) requirement under the prudentiality level $\omega$. For $\omega>0.5$, a higher value of $\omega$ leads to more prudent margin requirement which means a higher degree of risk aversion.

Alternatively, one can calculate $\omega$-expectile from the following identity [20]

$$
e_{\omega}(L)=E[L]+\frac{2 \omega-1}{1-\omega} \int_{e_{\omega}(L)}^{\infty}\left(l-e_{\omega}(L)\right) d F_{L}(l) .
$$

\section{The price-setting newsvendor model with evar criterion}

Since $\operatorname{EVaR}_{\omega}(L)$ is strictly increasing with respect to $\omega$, we choose $\omega \in(0.5,1)$ for more prudent and risk averse decisions, and $\omega \in(0,0.5)$ for less prudent and risk taker decisions. If we take $\omega=0.5$, the EVaR minimization model reduces to the classical price-setting newsvendor model. We derive the objective function EVaR from (10), so

$$
e_{\omega}(L)=E[L]+\frac{2 \omega-1}{1-\omega}\left(I_{1}+I_{2}\right)
$$

where

$$
\begin{aligned}
& I_{1}=\int_{0}^{q-a+b p}\left[(c-v) q-(p-v)(a-b p+t)-e_{\omega}(L)\right]^{+} d F_{\varepsilon}(t), \\
& I_{2}=\int_{q-a+b p}^{\infty}\left[s(a-b p+t)-(p+s-c) q-e_{\omega}(L)\right]^{+} d F_{\varepsilon}(t) .
\end{aligned}
$$

We need to investigate three separate cases

Case 1. $e_{\omega}(L) \leq(c-p) q$,

Case 2. $(c-p) q \leq e_{\omega}(L) \leq(c-v) q$,

Case 3. $e_{\omega}(L) \geq(c-v) q$. 
Case 1. For $I_{1},(c-v) q-(p-v)(t+a-b p) \geq e_{\omega}(L)$ since $0 \leq t \leq q-a+b p$. For $I_{2}, s(t+a-b p)-(p+s-c) q \geq e_{\omega}(L)$ since $q-a+b p \leq t$. So, both integrands are positive and

$$
\begin{gathered}
I_{1}=\int_{0}^{q-a+b p}\left[(c-v) q-(p-v)(a-b p+t)-e_{\omega}(L)\right] d F_{\varepsilon}(t), \\
I_{2}=\int_{q-a+b p}^{\infty}\left[s(a-b p+t)-(p+s-c) q-e_{\omega}(L)\right] d F_{\varepsilon}(t) .
\end{gathered}
$$

From (11),

$$
\begin{aligned}
& e_{\omega}(L)=E[L]+\frac{2 \omega-1}{1-\omega}\left(E[L]-e_{\omega}(L) F_{\varepsilon}(q-a+b p)-e_{\omega}(L)\left(1-F_{\varepsilon}(q-a+b p)\right) ;\right. \text { so } \\
& e_{\omega}(L)=E[L] .
\end{aligned}
$$

Our model is

$$
\begin{aligned}
& \min e_{\omega}(L) \\
& \text { subject to } \\
& \left\{\begin{array}{l}
e_{\omega}(L)=E[L], \\
p, q,(c-p) q-e_{\omega}(L), a / b-p \geq 0 .
\end{array}\right.
\end{aligned}
$$$$
\text { Problem } 1
$$

Case 2. Similarly, we get Problem 2 for Case 2 as following:

$$
\begin{aligned}
& \min e_{\omega}(L) \\
& \text { subject to } \\
& \left\{\begin{array}{l}
e_{\omega}(L)=E[L]+\frac{2 \omega-1}{1-\omega}\left(I_{1}+I_{2}\right), \\
p, q, e_{\omega}(L)-(c-p) q,(c-v) q-e_{\omega}(L), a / b-p \geq 0,
\end{array}\right.
\end{aligned}
$$

where

$$
\begin{aligned}
& I_{1}=\int_{0}^{\frac{(c-v) q-e \omega(L)}{p-v}-a+b p}\left[(c-v) q-(p-v)(a-b p+t)-e_{\omega}(L)\right] d F_{\mathcal{\varepsilon}}(t), \\
& I_{2}=\int_{\frac{e_{\omega}(L)+(p+s-c) q}{s}-a+b p}^{\infty}\left[s(a-b p+t)-(p+s-c) q-e_{\omega}(L)\right] d F_{\mathcal{\varepsilon}}(t) .
\end{aligned}
$$

Case 3. In a similar manner, one can get Problem 3 for Case 3 as following

$$
\begin{aligned}
& \min e_{\omega}(L) \\
& \text { subject to } \\
& \left\{\begin{array}{l}
e_{\omega}(L)=E[L]+\frac{2 \omega-1}{1-\omega}\left(I_{1}+I_{2}\right), \\
p, q, e_{\omega}(L)-(c-v) q, a / b-p \geq 0,
\end{array}\right. \\
& I_{1}=0, \\
& I_{2}=\int_{\frac{e_{\omega}(L)+(p+s-c) q}{s}-a+b p}^{\infty}\left[s(a-b p+t)-(p+s-c) q-e_{\omega}(L)\right] d F_{\varepsilon}(t) .
\end{aligned}
$$

To illustrate the proposed model, we present numerical results.

Example 1. This example is borrowed from [29]. Suppose the error distribution is Exponential with parameter $\lambda=0.1$ and $D(p, \varepsilon)=200-35 p+\varepsilon$. The unit salvage value $v=0.5$, the unit shortage cost $s=1$ and the unit ordering cost $c=1$. We solve our three problems separately and we get the optimal solutions from Problem 2. We present the risk taker and the risk neutral results in Table 1, and risk averse results in Table 2. Next, we generate 5000 error scenarios for exponential distribution and simulate profit function by using the corresponding optimal solutions $e_{\omega}(L), p^{*}, q^{*}$. Risk aversity increases when the risk parameter $\omega$ increases. According to the results of analysis and as expected, the standard deviation (volatility) of the profit function decreases and the gain-loss ratio increases when risk aversity increases. 


\begin{tabular}{|c|c|c|c|c|c|}
\hline$\omega$ & 0.1 & 0.2 & 0.3 & 0.4 & 0.5 \\
\hline$e_{\omega}(L)$ & -232.958 & -221.593 & -216.166 & -211.839 & -208.364 \\
\hline$p^{*}$ & 3.630 & 3,660 & 3.531 & 3.504 & 3.482 \\
\hline$q^{*}$ & 107.969 & 97.895 & 102.249 & 100.454 & 98.877 \\
\hline$E[\pi]$ & 203.912 & 206.421 & 207.430 & 207.941 & 208.101 \\
\hline$\sigma_{\pi}$ & 26.677 & 23.236 & 22.185 & 20.378 & 18.607 \\
\hline Gain/Loss & 0.577 & 0.744 & 0.974 & 1.173 & 1.327 \\
\hline
\end{tabular}

Table 1: Risk taker and the risk neutral results.

\begin{tabular}{|c|c|c|c|c|}
\hline$\omega$ & 0.6 & 0.7 & 0.8 & 0.9 \\
\hline$e_{\omega}(L)$ & -205.339 & -202.516 & -199.609 & -196.289 \\
\hline$p^{*}$ & 3.463 & 3.445 & 3.457 & 3.410 \\
\hline$q^{*}$ & 97.409 & 96.000 & 93.017 & 93.494 \\
\hline$E[\pi]$ & 207.980 & 207.604 & 206.747 & 206.063 \\
\hline$\sigma_{\pi}$ & 16.819 & 15.028 & 12.862 & 11.651 \\
\hline Gain/Loss & 1.541 & 1.816 & 2.189 & 3.182 \\
\hline
\end{tabular}

Table 2: Risk averse results.

\section{Concluding remarks}

In this paper, we use EVaR measure as an objective function to determine price-setting newsvendor solutions with risk considerations. We suppose that the random demand is the function of the selling price, especially the additive demand form is considered. In addition to the order quantity, the unit selling price is accepted as a decision variable in the classical newsvendor problem. When the newsvendor makes a decision with risk driven behavior, the optimal order quantity and price deviate from the classical expected profit maximizing quantity and price. Risk averse DMs dislike high volatility in their expected profits and they keep higher margin values. The primary contribution of this paper is that the aforementioned EVaR minimization model extends the classical price-setting newsvendor model through a one-parameter risk measure and facilitates a trade-off analysis between the capital to be held in reserve and the expected profit. When we use expectiles, the decision relies on both tails of the loss distribution and is easy to compute because of convexity. For risk averse newsvendors, another effective way to hedge against risk is to use pricing as a tool. Hopefully, we will extend our work for different demand models and provide sensitivitiy analysis for future research directions.

\section{Competing interests}

The authors declare that they have no competing interests.

\section{Authors' contributions}

All authors have contributed to all parts of the article. All authors read and approved the final manuscript.

\section{References}

[1] Ahmed, S., Çakmak, U., \& Shapiro, A. (2007). Coherent risk measures in inventory problems. European Journal of Operational Research, 182(1), 226-238. 
[2] Arcelus, F. J., Kumar, S., Srinivasan, G. (2012). Risk tolerance and a retailer's pricing and ordering policies within a newsvendor framework. Omega, 40(2), 188-198.

[3] Arıkan, E., \& Fichtinger, J. (2017). The risk-averse newsvendor problem under spectral risk measures: A classification with extensions. European Journal of Operational Research, 256(1), 116-125.

[4] Artzner, P., Delbaen, F., Eber, J. M., \& Heath, D. (1999). Coherent measures of risk. Mathematical finance, 9(3), $203-228$.

[5] Bellini, F. (2012). Isotonicity properties of generalized quantiles. Statistics \& Probability Letters, 82(11), 2017-2024.

[6] Bellini, F., \& Di Bernardino, E. (2015). Risk management with expectiles. The European Journal of Finance, 1-20.

[7] Chen, Y., Xu, M., \& Zhang, Z. G. (2009). A risk-averse newsvendor model under the CVaR criterion. Operations research, 57(4), 1040-1044.

[8] Chiu, C. H., Choi, T. M. (2010). Optimal pricing and stocking decisions for newsvendor problem with value-at-risk consideration. IEEE Transactions on Systems, Man, and Cybernetics-Part A: Systems and Humans, 40(5), 1116-1119.

[9] Choi, S., \& RuszczyńSki, A. (2008). A risk-averse newsvendor with law invariant coherent measures of risk. Operations Research Letters, 36(1), 77-82.

[10] Choi, S., Ruszczynski, A., \& Zhao, Y. (2011). A multiproduct risk-averse newsvendor with law-invariant coherent measures of risk. Operations Research, 59(2), 346-364.

[11] Dai, J., Meng, W. (2015). A risk-averse newsvendor model under marketing-dependency and price-dependency. International Journal of Production Economics, 160, 220-229.

[12] De Rossi, G. (2009). Staying ahead on downside risk. Optimizing Optimization: The Next Generation of Optimization Applications and Theory; Satchell, S., Ed, 143-160.

[13] Fichtinger, Johannes and Arikan, Emel, The Single Period Inventory and Pricing Problem with Spectral Risk Measures (January 19, 2011). Available at SSRN: https://ssrn.com/abstract=1611616 or http://dx.doi.org/10.2139/ssrn.1611616

[14] Föllmer, H., \& Weber, S. (2015). The axiomatic approach to risk measures for capital determination. Annual Review of Financial Economics, 7, 301-337.

[15] Gneiting, T. (2011). Making and evaluating point forecasts. Journal of the American Statistical Association, 106(494), 746-762.

[16] Gotoh, J. Y., \& Takano, Y. (2007). Newsvendor solutions via conditional value-at-risk minimization. European Journal of Operational Research, 179(1), 80-96.

[17] Jammernegg, W., \& Kischka, P. (2007). Risk-averse and risk-taking newsvendors: a conditional expected value approach. Review of Managerial Science, 1(1), 93-110.

[18] Katariya, A. P., Cetinkaya, S., \& Tekin, E. (2014). On the comparison of risk-neutral and risk-averse newsvendor problems. Journal of the Operational Research Society, 65(7), 1090-1107.

[19] Kuan, C. M., Yeh, J. H., \& Hsu, Y. C. (2009). Assessing value at risk with care, the conditional autoregressive expectile models. Journal of Econometrics, 150(2), 261-270.

[20] Newey, W. K., \& Powell, J. L. (1987). Asymmetric least squares estimation and testing. Econometrica: Journal of the Econometric Society, 819-847.

[21] Özler, A., Tan, B., \& Karaesmen, F. (2009). Multi-product newsvendor problem with value-at-risk considerations. International Journal of Production Economics, 117(2), 244-255.

[22] Sarykalin, S., Serraino, G., \& Uryasev, S. (2008). Value-at-risk vs. conditional value-at-risk in risk management and optimization. Tutorials in Operations Research. INFORMS, Hanover, MD, 270-294.

[23] Wu, M., Zhu, S. X., \& Teunter, R. H. (2013). Newsvendor problem with random shortage cost under a risk criterion. International Journal of Production Economics, 145(2), 790-798.

[24] Wu, M., Zhu, S. X., Teunter, R. H. (2014). A risk-averse competitive newsvendor problem under the CVaR criterion. International Journal of Production Economics, 156, 13-23.

[25] Xu, M. (2010). A price-setting newsvendor model under CVaR decision criterion with emergency procurement. Journal of Systems Science and Systems Engineering, 19(1), 85-104.

[26] Xu, M., \& Chen, F. Y. (2007, December). Tradeoff between expected reward and conditional value-at-risk criterion in newsvendor models. In 2007 IEEE International Conference on Industrial Engineering and Engineering Management (pp. 1553-1557). IEEE.

[27] Xu, M., \& Li, J. (2010). Optimal decisions when balancing expected profit and conditional value-at-risk in newsvendor models. Journal of Systems Science and Complexity, 23(6), 1054-1070. 
[28] Yang, J. (2017). Monotone trends in inventory-price control under time-consistent coherent risk measure. Operations Research Letters, 45(3), 293-299.

[29] Yao, L., Chen, Y. F., Yan, H. (2006). The newsvendor problem with pricing: extensions. International Journal of Management Science and Engineering Management, 1(1), 3-16.

[30] Zhang, D., Xu, H., \& Wu, Y. (2009). Single and multi-period optimal inventory control models with risk-averse constraints. European Journal of Operational Research, 199(2), 420-434. 